\title{
SLOPE MONITORING STUDY USING SOIL MECHANICS PROPERTIES AND 4-D ELECTRICAL RESISTIVITY TOMOGRAPHY METHODS
}

UDC 624.131.43:550.837

\author{
Andy Anderson Bery \\ Geophysics Section, School of Physics, Universiti Sains Malaysia, \\ Penang, Malaysia.
}

In past applications of electrical resistivity survey, users commonly focussed on the Earth's subsurface to locate water table, bedrock, etc. Nowadays, this electrical resistivity method is used by engineers for shallow subsurface investigations. This paper presents the integration of soil mechanics properties with electrical resistivity tomography methods and recommends the proper selection of resistivity array (survey planning) and appropriate inversion constraint parameters (data processing) that are able to deliver optimum resistivity tomography model results. This study identifies the empirical correlations of the soil's properties such as shear strength parameters, moisture content, void ratio, porosity, saturation degree, and Atterberg's limits with the electrical resistivity values (resistivity tomography models). A total of 11 undisturbed clayey sand soil samples was collected at different distances, depths, and times and were tested under both infield and laboratory conditions during the slope monitoring period. The soil mechanics properties of the soil samples were obtained right after the electrical resistivity survey was made. It is shown that the electrical resistivity values are greatly influenced by the soil mechanics properties. Thus, the electrical resistivity (4-D) survey using the optimized WennerSchlumberger array (high data density) is capable of reliably enhancing the conventional outcome of the Earth's subsurface investigation.

\section{Introduction}

The need to analyze the soil's behavior has emerged around the world and is often a result of critical problems such as failures of foundation and landslides. Soil mechanics is evolving into a different branch of engineering due to the fact that soils have special properties or characteristics that are distinctive. It is crucial to know and understand the soil's behavior because it changes when mixed with other substances such as water (rain). Many methods have been used by researchers to study the soil. These include resonance methods [1], analytical models [2], and fiber materials (papyrus) [3]. Knowledge of the soil's characteristics is important because it is closely related to construction and economic purposes. The electrical resistivity technique requires measurement of the potential difference and current at the Earth's surface. This technique has long been used in geophysical surveying for commercial purposes. The electrical resistivity survey method is one of the most common and oldest geophysical investigation methods [4]. The use of this geophysical method is closely related to the chemistry properties of the Earth's subsurface. This geophysical method has some limitations that should be taken into considerations. For example, different types of arrays will produce different results over the same target or Earth's structure.

Translated from Osnovaniya, Fundamenty i Mekhanika Gruntov, No. 1, p. 20, January-February, 2016. 
This paper presents the two main subject matters that must be considered for geotechnical works such as natural slope monitoring [5-7], buried bunker [8], meteorite impact [9, 10], geothermal [11], hydrogeological [12], and mineral exploration [13] surveys. However, studies that relate geotechnical properties to electrical resistivity data are still rare [14]. The objective of this study is to identify the correlation between electrical resistivity values (resistivity tomography model) and other properties such as shear strength parameters, moisture content, void ratio, porosity, saturation degree, and Atterberg's limits. In addition, this study was conducted at a natural slope area with the application of time-lapse (4-D) electrical resistivity tomography to investigate the changes of the soil's properties during the slope monitoring period. It is shown that the integration of the geophysical and geotechnical engineering methods is capable of producing optimum information on the soil mechanics properties at the Earth's subsurface.

\section{Electrical Resistivity Survey and Geotechnical Laboratory Tests}

We used the infield time-lapse electrical resistivity (4-D) method and performed several geotechnical laboratory tests. First of all, the best array among all the common electrical arrays was chosen. The selection of the best array must rely on the structure to be mapped, the sensitivity of the equipment (resistivity meter), and the surrounding noise level. Among the considerations for array selection are the signal strength, the sensitivity of the array to both horizontal and vertical changes in resistivity pattern, the horizontal data coverage, and the depth of investigation [15]. In this study, the Wenner-Schlumberger array is selected after comprehensive considerations. The Wenner-Schlumberger array is a hybrid array from the combination of the Wenner-Alpha and Schlumberger arrays [15-17]. The signal strength of this array is higher compared to Dipole-Dipole and Pole-Dipole arrays. The depth of investigation (penetration) is medium, and it is less sensitive to surrounding noise. Moreover, the horizontal data coverage of the Wenner-Schlumberger array is wider than the Wenner-Alpha array. A single survey line of the electrical resistivity survey was conducted at Minden, Universiti Sains Malaysia using ABEM SAS4000 resistivity meter. The infield survey was conducted with a minimum electrode spacing of $0.5 \mathrm{~m}$ to obtain a good data quality as there will be decreasing signal strength with increasing distance from the electrodes at ground surface. It helps to improve the spatial resolution of model resistivity with tomography results. The length of the survey line is $20 \mathrm{~m}$. The electrical resistivity survey was repeated along the same line and the same electrode location at different times during the slope monitoring study. A total of 41 stainless-steel electrodes was used for the electrical resistivity survey. All the electrodes were connected through two resistivity cables that are connected by jumper cables. The electrode selector (ES10-64) is connected to the SAS4000 unit for comprehensive data acquisition. The WennerSchlumberger array used in this survey is an optimized array with maximum data points of 665 . This hybrid array is suitable for high-resolution electrical resistivity tomography. The data obtained from the field measurement was transferred and converted using the SAS4000 utilities software to obtain resistivity values to present the geometry of the electrical resistivity survey. The data were processed and analyzed using the RES2DINV program to provide an inverse model resistivity with topography. Some of the appropriate constraint parameters in the processing works are shown in Table 1. These constraint parameters are useful in getting an optimum of model resistivity with topography results. These constraint parameters were set based on the target to be mapped and the noise level of the data set.

Finally, three important parameters ( $x$-distance, $y$-depth, and $z$-resistivity values) can be used in correlation with the soil mechanics properties from the geotechnical laboratory tests. A total of 11 clayey sand soil samples at different distances, depths, and times were collected and tested under both infield and laboratory conditions during the slope monitoring period. The study area was located at Universiti Sains Malaysia, Penang. The major portion of Penang is underlain by igneous rocks classified as granites type. 
TABLE 1

\begin{tabular}{|l|c|}
\hline Inversion constraint parameters & Value / Decision \\
\hline Initial damping factor & 0.05 \\
Minimum damping factor & 0.01 \\
Factor to increase damping factor & 1.10 \\
Optimize the damping factor & Yes \\
Forward modeling method & Finite-difference \\
Least-squares constraint & L1 Norm \\
\hline
\end{tabular}

a

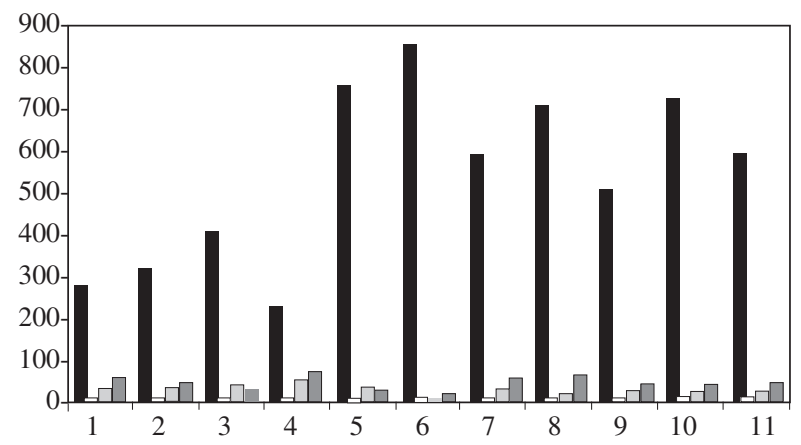

b

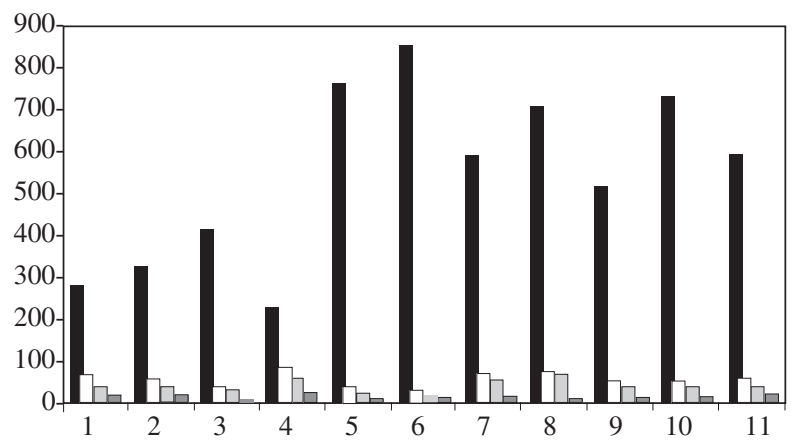

Fig. 1. Correlation of electrical resistivity values $\rho(\mathbf{m})$ with: a) the soil shear strength parameters ( $\square$ - effective cohesion $c^{\prime}, \mathrm{kN} / \mathrm{m}^{2} ; \square$ - internal friction angle $\varphi^{\prime}$, deg.; $\square$ - moisture content $W, \%$ ); and b) Atterberg's limits ( $\square$ - liquid limit $W_{L}, \%$; $\square$ - plastic limit $W_{p}, \%$; $\square$ - plasticity index $\left.P_{I}, \%\right)$ for the 11 clayey sand soil samples collected during the slope monitoring period.

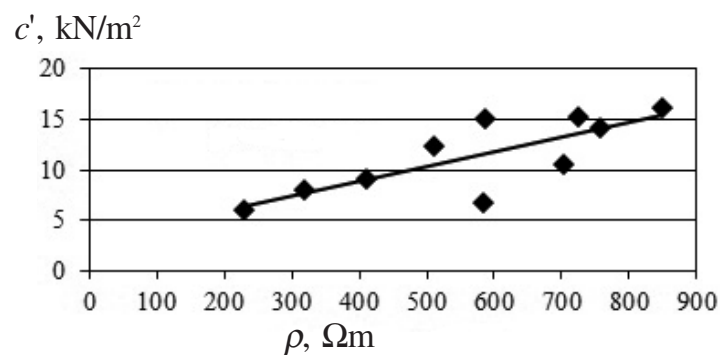

$\mathrm{b}$

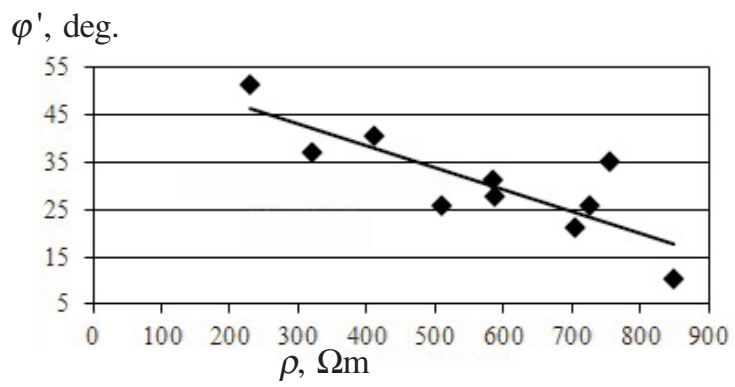

Fig. 2. The empirical correlation between resistivity $\rho$ and a) effective cohesion $c^{\prime}$; and b) internal friction angle $\varphi^{\prime}$ of undisturbed clayey sand soil samples.

\section{Results and Discussion}

This chapter presents the results of an environmental monitoring study conducted in the study area for five months. The study consists of a combination of two different methods: the geophysical and the geotechnical engineering. In this study, the undisturbed clayey sand soil sample was taken manually from the study area at several distances and depths for the direct shear test. All the results presented in Fig. 1 are based on the infield resistivity method and their soil mechanics properties from several laboratory tests.

The empirical correlation between the resistivity $\rho$ and the undisturbed soil's effective cohesion for clayey sand soils $c^{\prime}=0.014 \rho+3.156$, in which the regression coefficient $R^{2} \approx 0.651$ (Fig. 2a). The relation shows that the higher the resistivity values, the higher the effective cohesion values. This is associated with the density and compression of the clayey sand soils. 


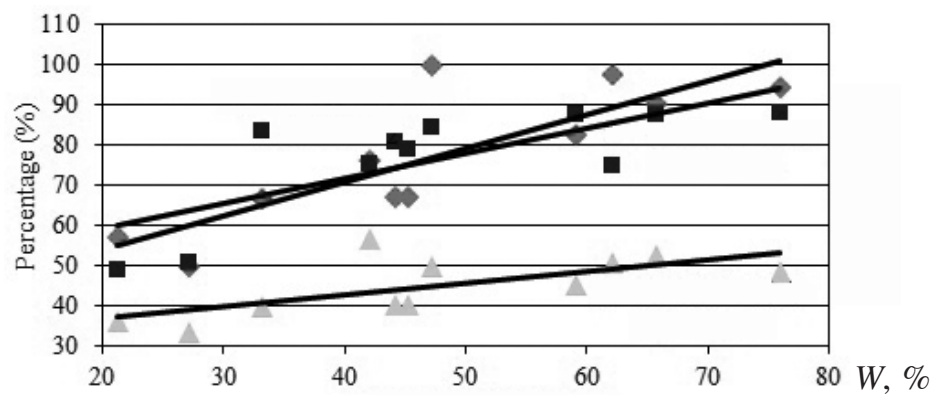

Fig. 3. Empirical correlation of moisture content $W$ with: $\bullet$ ) void ratio $e=0.845 W+36.63$, $\left.R^{2}=0.692 ; \Delta\right)$ porosity $n=0.317 W+28.41, R^{2}=0.724 ;$ and a) saturation degree $S=0.629 \mathrm{~W}+46.30, R^{2}=0.570$ of clayey sand soil samples.

The empirical correlation between the resistivity $\rho$ and the internal friction angle $\varphi^{\prime}$ for undisturbed clayey sand soils is determined to be $\varphi^{\prime}=-0.041 \rho+53.80$, and $R^{2} \approx 0.652$. This relation indicates that the strength of clayey sand soil is governed by the angularity of sand particles and the presence of water (low resistivity) in clayey sand soils (Fig. 2b).

The empirical correlations between moisture content and void ratio, porosity, and degree of saturation of clayey sand soil are shown in Fig. 3. It is shown that the void ratio and porosity play a role in fluid conductivity control (FCC), also known as the ability of water to move through the soil's particles. The saturation degree shows the amount of water present in clayey sand soils.

This study is focussed on the relationship between electrical resistivity and soil properties using the undisturbed and disturbed clayey sand soil condition.

Fig. 4 shows the percentage change in the model resistivity with topography at the Earth's subsurface in the study area during the slope monitoring period (February to May). The time-lapse resistivity tomography (4-D) method in this study used January's data set acts as reference. All the models for the time-lapse resistivity study were able to produce an absolute error of less than 5\%. This small absolute error signifies good quality data sets. From the model resistivity with tompography, the higher negative percentage change shows that the region is suspected to be a weak zone and the soils is mixed up with moisture content (decreasing in resistivity values). These zones or regions are closely related to increase in moisture content in the clayey sand soils. The bond between the soil's particles is weakened by the presence of moisture content, and thus the resistivity values decrease.

\section{Conclusion and Recommendation}

The empirical correlations between the electrical resistivity values and the soil mechanics properties are beneficial in providing the characteristics of the soil within the slope monitoring period. The objective of this study is successfully achieved through the demonstration of the characteristics specifically of the clayey sand soil's behavior. All the empirical correlations showed that the soil mechanics properties greatly influence the electrical resistivity values. The electrical resistivity values were influenced by the changes in chemical properties (moisture content), which in turn related to the pattern of current flow through the soil's particles. In addition, the application of the time-lapse electrical resistivity tomography (4-D) method using an optimized Wenner-Schlumberger array, coupled with appropriate inversion constraint parameters, has successfully produced good correlations between electrical resistivity values and soil mechanics properties during the slope monitoring period. The establishment of these empirical correlations can be improved by increasing the number of soil samples for the engineering laboratory tests. It is thus deduced that good estimation of soil mechanics properties can be applied and verified from the results of the electrical resistivity tomography model. 


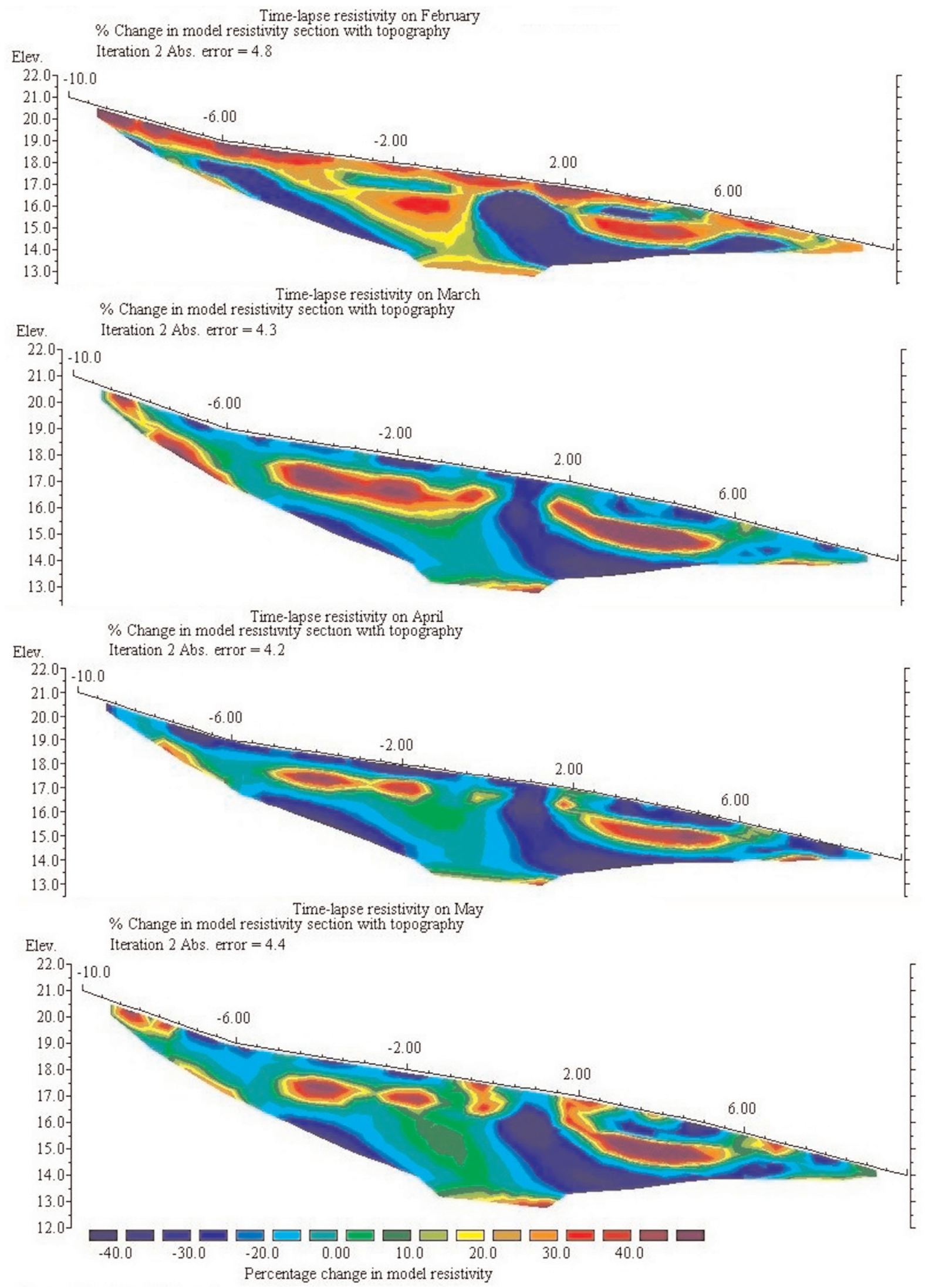

Fig. 4. Time-lapse resistivity (4-D) results showing the changes of electrical resistivity distribution in slope monitoring along the same study line (same electrodes location) over different times (from February to May). 


\section{REFERENCES}

1. L. R Stavnitser and G. A. Nikitaeva, "Resonance method of determining the damping characteristics of soils," Soil Mech. Found. Eng., 45, 9-12 (2008).

2. D. M. Shapiro and A. A. Tarasov, "Analytical models of beds of inclined structures and retaining walls of reinforced soils," Soil Mech. Found. Eng., 51, 181-187 (2014).

3. A. Adili, R. Azzam, G. Spagnoli, and J. Schrader, "Strength of soil reinforced with fiber materials (Papyrus)," Soil Mech. Found. Eng., 48, 241-247 (2012).

4. J. M. Reynolds, An introduction to applied and environmental geophysics, 2nd edition. John Wiley \& Sons, England (2011).

5. A. A. Bery, "Slope monitoring study using time-lapse resistivity tomography and engineering soil's characterization methods," World Appl. Sci. J., 32, 1364-1368 (2014).

6. A. A. Bery, and R. Saad, "Enhanced horizontal and vertical resolution in 4-D electrical resistivity for environmental slope study in Penang Island," Appl. Mech. Mater., 661, 73-80 (2014).

7. A. A. Bery R. Saad, Y. C. Kiu, and N. A. Kamaruddin, "High resolution time-lapse resistivity tomography with merging data levels by two different optimized resistivity arrays for slope monitoring study," Electron. J. Geotech. Eng., 19, 503-509 (2014).

8. A. A. Bery and R. Saad, "Merging data levels using two different arrays for high resolution resistivity tomography," Electron. J. Geotech. Eng., 18, 5507-5514 (2013).

9. M. Jinmin, R. Saad, M. M. Saidin, Y. C. Kiu, and A. A. Bery, "Second stage subsurface study of meteorite impact crater at Bukit Bunuh, Malaysia using 2D electrical resistivity survey," Electron. J. Geotech. Eng., 18, 1199-1204 (2013).

10. R. Saad, M. Mokhtar Saidin, N. M. Muztaza, N. A. Ismail, N. E. H. Ismail, A. A. Bery, and E. T. Mohamad, "Subsurface study using 2-D resistivity imaging method for meteorite impact at Bukit Bunuh, Perak," Electron. J. Geotech. Eng., 16, 1507-1513 (2011).

11. M. G. Drahor, M. A. Berge, O. Bakak, O. and O. Caner, "Electrical resistivity tomography monitoring studies at Balcova (Turkey) geothermal site," Near Surf. Geophys., 12, 337-350 (2014).

12. L. M. Page, "Use of the electrical resistivity method for investigating geological and hydrogeological conditions in Santa Clara Country, CA," Ground Water, 6, 31-40 (1968).

13. P. Baurman, "2-D resistivity surveying for hydrocarbon-a primer," CSEG Recorder April 2005, 25-33 (2005).

14. P. Cosenza, E. Marmet, F. Rejiba, Y. Jun Cui, A. Tabbagh, and Y. Charlery, "Correlation between geotechnical and electrical data: a case study at Garchy in France," J. Appl. Geophys., 60, 165-178 (2006).

15. A. A. Bery, "Depth analysis for sensitivity pattern section in the electrical resistivity using the 2D computerized modeling method," Electron. J. Geotech. Eng., 19, 9389-9400 (2014).

16. A. A. Bery, R. Saad, I.N.E. Hidayah, I.N. Azwin, and M. Saidin. "Enhancement in resistivity resolution based on the data sets amalgamation technique at Bukit Bunh, Perak, Malaysia," IOP Conf. Series: Earth and Environmental Science, 23, 012009, (2015).

17. A. A. Bery. "2-D mechanics models fabrication using electrical resistivity tomography method for slope monitoring study," Int. J. Appl. Eng. Res., 10, 37175-37181 (2015). 